\title{
Acute glaucoma following vitrectomy and silicone oil injection
}

\author{
LILLY ZBOROWSKI-GUTMAN, GIORA TREISTER, NAVA NAVEH, \\ VARDA CHEN, AND MICHAEL BLUMENTHAL \\ From the Goldschleger Eye Institute, Chaim Sheba Medical Center, Tel Hashomer, 52621, Israel
}

\begin{abstract}
SUMmaRY Three cases are described of acute glaucoma following vitrectomy and silicone oil injection in proliferative vitreous retinopathy. The first case developed silicone-induced pupillary block in a phakic eye. Cases 2 and 3 developed elevated pressure in aphakic eyes with deep anterior chambers. Cases 1 and 3 were treated by laser iridectomy. Case 2 was treated by removal of silicone. The pathogenesis and treatment of these problems are discussed.
\end{abstract}

In recent years there has been increasing success in the surgical treatment of severe cases of proliferative vitreous retinopathy (PVR) by a combination of vitrectomy and silicone oil injection..$^{1-3}$ Attention has recently been drawn to the risk of aphakic pupillary block glaucoma. ${ }^{+}$The possibility of direct block of the trabeculum has also been proposed. ${ }^{5}$

We present three cases of acute glaucoma in phakic and aphakic eyes following silicone oil injection $(1000 \mathrm{cs})$. The silicone itself was directly implicated in the pressure elevation. These cases demonstrate the various ways that silicone oil can disrupt aqueous humour dynamics in the anterior chamber.

\section{Case reports}

CASE 1

A 49-year-old man was referred to us for vitrectomy for severe PVR in the right eye. Ten months earlier he had undergone a buckling procedure for a large retinal hole. The retina was initially attached but later became detached again and the vitreous became organised. The left eye had undergone focal laser treatment for a retinal hole. At our initial examination the visual acuity was RE hand motion and LE 6/9. Intraocular pressure was $15 \mathrm{mmHg}$ in both eyes and the angles were wide open on gonioscopy. The lenses were clear. On 18 June 1985 he underwent pars plana vitrectomy, membranectomy, and silicone oil injection with placement of an encircling band. During the procedure a small amount of silicone was noted to enter the anterior chamber, possibly owing

Correspondence to Lilly Zborowski-Gutman, MD, Tagore 40, Tel Aviv, Israel. to a small zonulysis. The following day the anterior chamber was flat peripherally. The pupil was middilated, with silicone bulging through, forming a shallow chamber centrally. The posterior chamber was very deep (about $2 \mathrm{~mm}$ ) and completely filled with silicone (Fig. 1). The IOP was $35 \mathrm{mmHg}$ and the angle was closed.

A YAG laser iridotomy was performed in the midperiphery at the 3 o'clock position with immediate and dramatic deepening of the entire anterior chamber. The IOP dropped to $25 \mathrm{mmHg}$ and later to $14 \mathrm{mmHg}$. The angle opened completely. A bulge of silicone remained in the pupil and the retina was attached. Five months after the silicone oil injection the lens became cataractous and he underwent an uneventful extracapsular cataract extraction with posterior chamber intraocular lens. Eleven months after the initial procedure the vision was $6 / 90$, the retina was attached, and the intraocular pressure remained in the normal range without treatment.

\section{CASE 2}

A 57-year-old woman had undergone two retinal buckling procedures in her left eye. She was aphakic and highly myopic in both eyes and suffered from diabetes mellitus. On 3 March 1985 she underwent pars plana vitrectomy with silicone oil injection because of a severe PVR of the left eye.

Postoperatively the anterior chamber was filled with silicone but the IOP was normal. Two months postoperatively she presented with severe pain in the operated eye. The IOP was $64 \mathrm{mmHg}$, and the anterior chamber was deep and filled with silicone. The pupil was dilated, and angle structures could not 


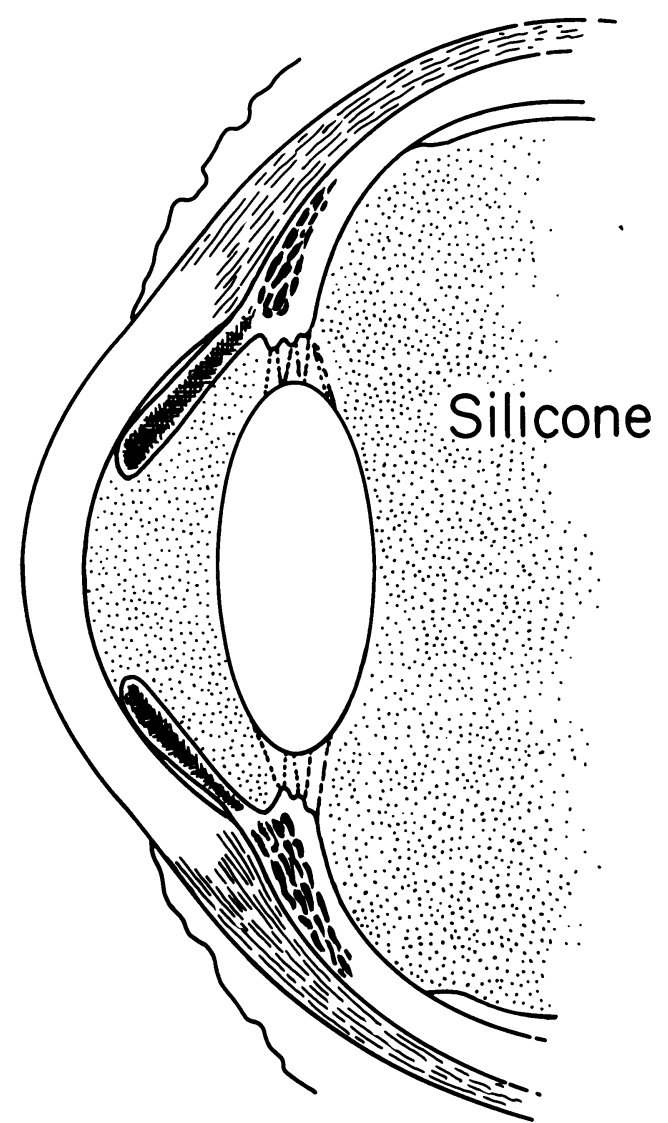

Fig. 1 Case 1, one day after operation. Note the silicone filling the posterior chamber and the extreme anterior displacement of the iris.

be seen. Conservative measures were unsuccessful in lowering the pressure, and she then underwent a partial drainage of the silicone via a limbal paracentesis. The IOP dropped to $8 \mathrm{mmHg}$. One month later, she again presented with an IOP of $64 \mathrm{mmHg}$ and silicone oil filling the anterior chamber. Treatment was conservative and included lying prone.

The IOP dropped to $12 \mathrm{mmHg}$, and the silicone oil remained posterior to the iris. One year later the retina remained attached and the IOP was controlled with timolol and acetazolamide.

CASE 3

A 68-year-old woman was referred to us after two unsuccessful retinal buckling procedures in her left eye. At the initial examination her acuity was RE $5 / 7.5$ and LE counting fingers. The IOP was RE 18 $\mathrm{mmHg}, \mathrm{LE} 10 \mathrm{mmHg}$. The left eye had severe PVR and a vitreous haemorrhage. On 24 June 1984 she underwent a pars plana vitrectomy with silicone oil injection. On 26 February 1985 she underwent intracapsular cataract extraction via a limbal incision with membranectomy and repeat silicone injection because of progressive traction detachment. The retina was reattached and her vision slowly improved to 6/30. In March 1985 the IOP began to rise and responded initially to medical therapy. The pressure became refractory to treatment and was as high as 42 $\mathrm{mmHg}$. On 4 July under timolol and acetazolamide the IOP was RE $17 \mathrm{mmHg}$ and LE $28 \mathrm{mmHg}$. The anterior chamber was deep and entirely filled by silicone. Gonioscopy revealed peripheral anterior synechiae in the superior quadrant and an angle open to the scleral spur over the remaining $270^{\circ}$. The cup/disc ratio was OD $0 \cdot 2-0 \cdot 3$ and OS $0 \cdot 7$. A YAG q-switched laser iridotomy was performed at the 5 o'clock position. Thirty minutes later only a bulge of silicone was seen through the pupil and the remainder of the chamber was filled with aqueous. The appearance of the angle did not change, but the IOP was again amenable to medical therapy. Ten months after the laser iridectomy her vision was $6 / 60$ and the IOP $19-24 \mathrm{mmHg}$ under treatment with timolol and acetazolamide.

\section{Discussion}

Silicone oil is highly viscous and has a specific gravity less than water. The use of silicone has been shown to be effective in the treatment of PVR and sealing of retinal holes. ${ }^{1-3}$ These same properties constitute a danger to aqueous humour dynamics in the anterior segment. As Ando has described in aphakic eyes, pupillary block can occur when the viscous silicone obstructs the pupil and an iridectomy, which is typically superiorly located. The problem will be alleviated by an iridectomy (surgical or laser) that is in the lower quadrant. The silicone, being lighter than water, rises in the intraocular space and obstructs the upper iridectomy. A small amount of fluid always remains in the inferior vitreous space.

Our case 1 developed a similar problem but in a phakic eye. To our knowledge this has not been previously described. During surgery there was a small zonulysis that allowed access of the silicone to the anterior chamber. Postoperatively this anterior flow of silicone apparently led to pupillary block. The striking anatomical feature of this case was the extremely deep posterior chamber and the almost flat anterior chamber, which made the treatment more difficult. The case bears some resemblance to the 'anterior pupillary block' syndrome described by Lazar," where the impediment to normal aqueous flow was an iridocorneal adhesion. If silicone oil is noted in the anterior chamber during surgery in the 
phakic eye, it is recommended that a 6 o'clock iridectomy be performed as soon as possible.

In cases 2 and 3 the IOP rose in aphakic eyes with open angles on gonioscopy and deep chambers filled with silicone. It seemed that the silicone was blocking the trabecular meshwork by direct tamponade and that the best treatment would be to extract some of the silicone. This of course revives the risk of retinal detachment. Furthermore, as seen in case 2, this method of treatment may not be entirely effective. After extraction more silicone may flow forwards and trigger the same cycle of pressure elevation.

The true aetiology of pressure elevation in these two cases was a combination of pupillary block and direct obstruction.

Even when maximal amounts of silicone are injected, a small amount of fluid remains inferiorly as described above. As aqueous is produced, this pocket enlarges and pushes the silicone further and further through the pupil until the entire anterior chamber and pupillary space is filled (Fig. 2). No aqueous can now reach the anterior chamber, and the pressure rises precipitously. The lower iridectomy allows at least a portion of the fluid to flow forward and find a level. Simultaneously some of the silicone flows posteriorly through the pupil (Fig. 3). The pupillary block is bypassed by the iridectomy, and the posterior movement of the silicone alleviates the direct block of the trabecular meshwork.

In their series Leaver and Grey ${ }^{7}$ described pressure elevations in 15\% (14/93) of their cases. In nine of 14 eyes this was associated with numerous fine globules of silicone in the superior angle. They did not describe any cases of pupillary block. Similarly Haut et al. ${ }^{8}$ noted elevated IOP in 23 of 200 cases of silicone injection, mostly in aphakes. Twelve cases were considered angle closure, but they did not specify whether from peripheral anterior synechiae or

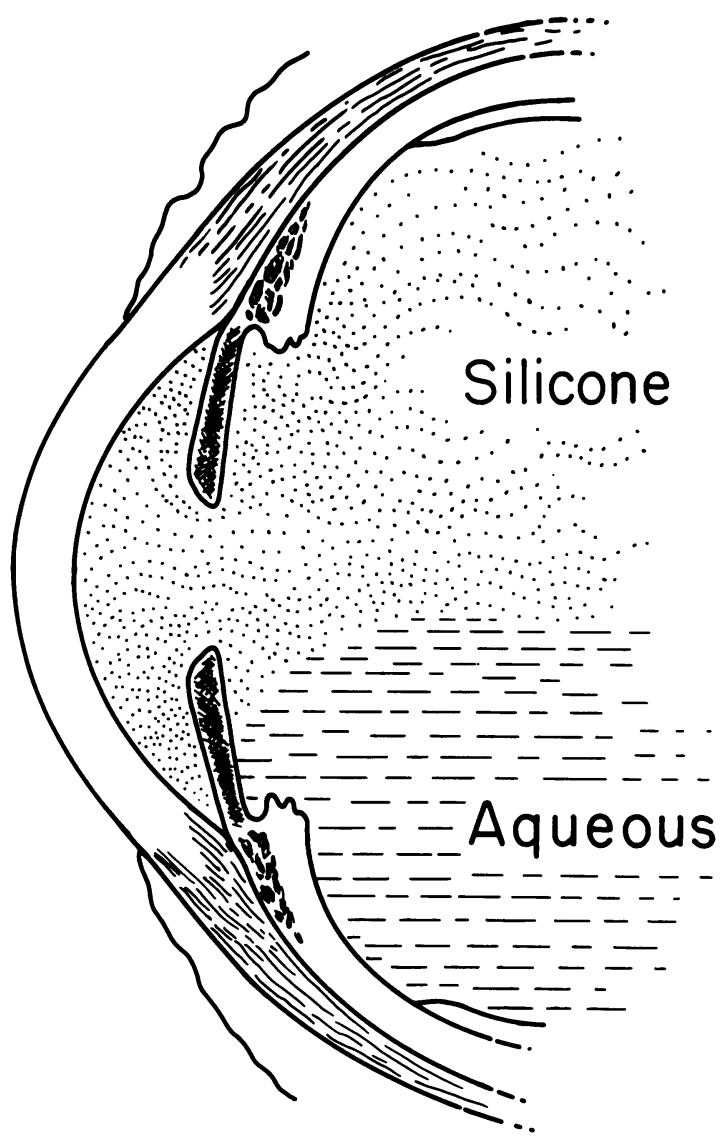

Fig. 2 Aqueous has sunk to the lower vitreal space. The silicone oil completely fills the remaining vitreous and pupillary space and the entire anterior chamber. The aqueous has no access to the trabecular meshwork.

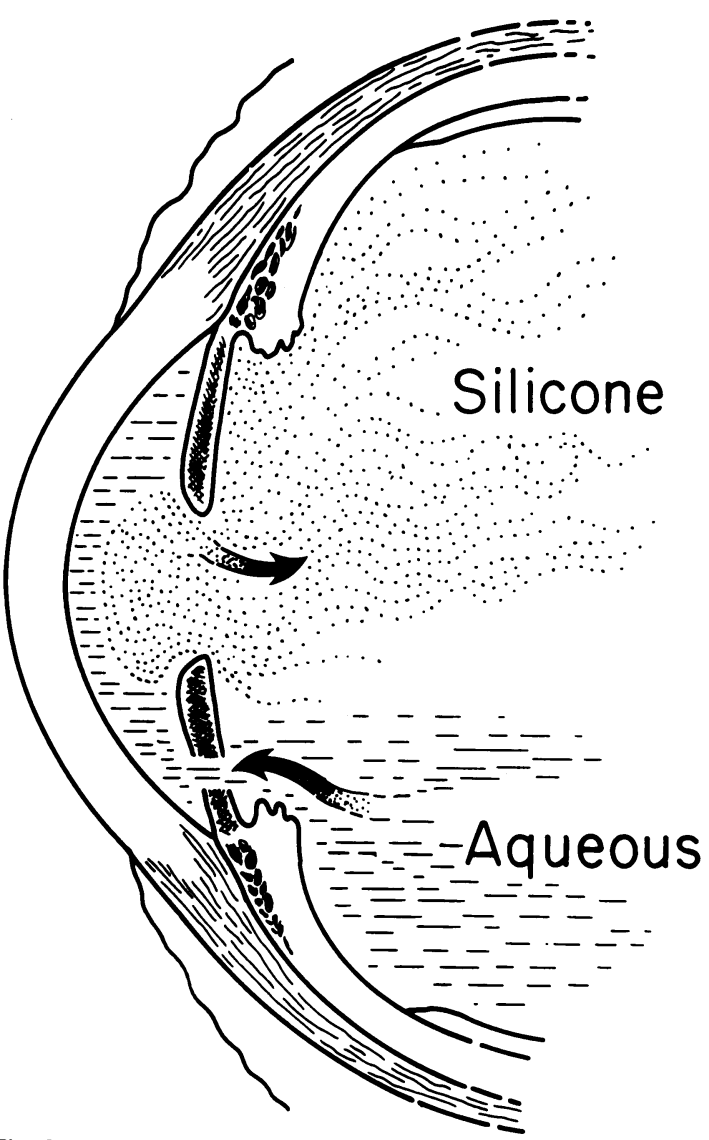

Fig. 3 After an inferior iridectomy the trapped aqueous flows anteriorly. This allows some silicone oil to flow posteriorly through the pupil. Both the pupillary block and the direct block of the trabeculum are alleviated. 
pupillary block. In some of their cases fine silicone emulsion bubbles were seen. We have noted silicone bubbles in a few of our silicone cases but not in the cases herein described.

Silicone oil carries a significant risk for causing pupillary block glaucoma both in phakic and in aphakic eyes. Moreover, in cases where the pupillary block component is not initially suspected because of a deep anterior chamber, a strategically placed lower iridectomy can obviate more complicatèd procedures. This aspect of silicone oil complication has not been stressed in previous reports.

Pars plana vitrectomy with silicone oil injection is being performed with increasing frequency and success. Consequently the attendant complication of pupillary block glaucoma will be seen more often in both the immediate and long-term postoperative periods.
References

1 Scott JD. Treatment of massive vitreous retraction. Trans Ophthalmol Soc UK 1975; 95: 429-32.

2 Grey RHB, Leaver PK. Results of silicone oil injection in massive preretinal retraction. Trans Ophthalmol Soc UK 1977; 97: 238-41.

3 Zivognovic R, Mertens PA, Peperkamp E. Fluid liquid silicone in amotio surgery. Klin Monatsbl Augenheilkd 1982; 181: 444-52.

4 Ando F. Intraocular hypertension resulting from pupillary block by silicone oil. Am J Ophthalmol 1985; 99: 87 .

5 Phelps CD. Glaucoma associated with retinal disorders. In: Ritch R, Shields MB, ed. The secondary glaucomas. St Louis: Mosby, 1982.

6 Lazar M, Godel V. Anterior pupillary block. Acta Ophthalmol (Kbh) 1981; 59: 221-4.

7 Leaver PK, Grey RH, Garner A. Silicone oil injection in the treatment of massive preretinal retraction. II. Late complications in 93 eyes. Br J Ophthalmol 1979; 63: 361-7.

8 Haut J, Ullern M, Chermet M, Van Effentere G. Complications of intraocular silicone and their possible treatment. Ophthalmologica 1980; 180: 29-35.

Accepted for publication 20 November 1986. 